\title{
A NEW PEDAGOGICAL TOOL FOR THE TRAINING OF ENGINEERS IN ANALOG ELECTRONICS
}

Constantin, N G; Ayissi Eyebe, G; Nerguizian, V.

Electrical Engineering Department, École de technologie supérieure, Quebec Canada nicolas.constantin@etsmtl.ca

\section{INTRODUCTION}

In this communication, we present a new pedagogical tool intended for the training of undergraduate engineers in analog electronics. Though currently considered to be adapted for the electrical engineering curriculum at the École de technologie supérieure (ÉTS) - Université du Québec, we believe that the underlying approach should be applicable and beneficial to any electrical engineering program.

The broad range of courses in the electrical engineering curriculum at the ÉTS, together with the industrial training or internship activities, inevitably create long interruption periods between the complementary courses in the student's progress towards the analog electronics specialty. Yet, the analog and high frequency electronics specialty requires a considerable effort from the student in the synthesis of the concepts and circuit techniques covered through the various courses that deal with analog circuits and systems, besides simply the completion of these courses individually. The proposed pedagogical tool has been the object of an institutional PSIRE research funding (from the French: Programme de Support Institutionnel à la Recherche et à l'Enseignement), and is aimed at supporting the student's efforts all along his analog course curriculum.

\section{METHODS}

The student's knowledge in analog electronics would benefit significantly from a continued training during any interruption period between complementary courses throughout the undergraduate and graduate electrical engineering program. Accordingly we are developing an innovative and motivating pedagogical tool at the ÉTS. The approach under study implies: (i) the development of on-line pedagogical tools for training in analog electronics and which are easily accessible by the students via the intranet sites of the ÉTS, (ii) through these tools, the means for efficient review and self-evaluation on analog electronics material on a continuous basis, while being appealing for its application to all related concepts covered in other courses, and (iii) enabling an efficient interaction with professors when used for review at the beginning or during any related course.

As shown in Figure 1, the approach uses a simple and widely known interface (e.g. Excel ${ }^{\mathrm{TM}}$ ) accessible via on-line sessions on the university's intranet, and that allows the student interface with two modules (not directly accessible by the student) that perform analyses, computations and evaluations.

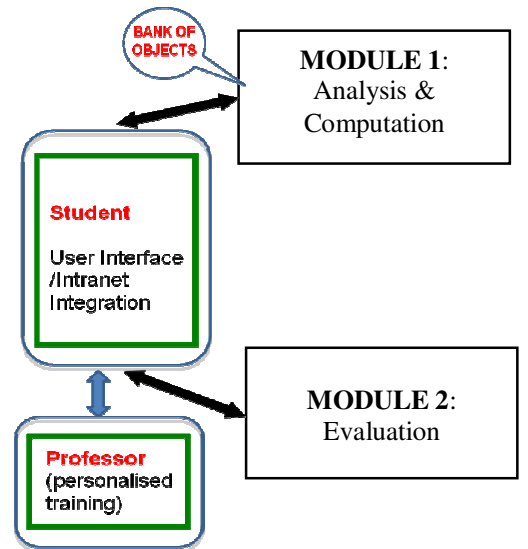

Figure 1: Structure of the proposed on-line pedagogical tool.

The first module (Figure 2) contains a bank of objects in the form of electronic schematics, graphs, review topics and questions that are accessed through the user interface (Figure 1) and presented to the student. This module does the necessary analysis and computation required to validate the answers from the user, and generates relevant topic review information that helps the student at various levels of difficulty, depending on the question selected. It also provides the correct answer and explanations when the student gives a wrong answer. The analyses and computations are carried out with circuits and systems analytical formulations, or by interfacing with a circuit simulator (e.g. Microcap ${ }^{\mathrm{TM}}$ or PSPICE).

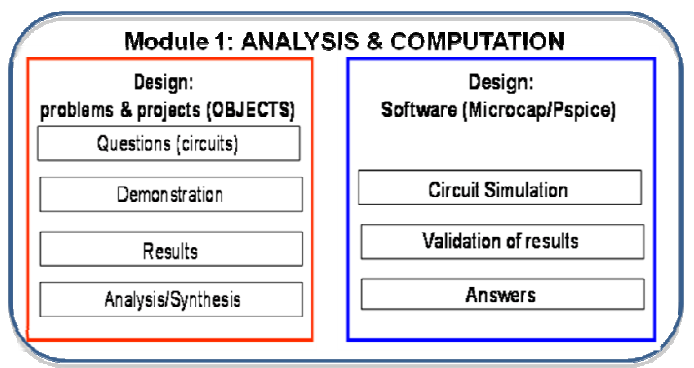

Figure 2: Analysis and computation module.

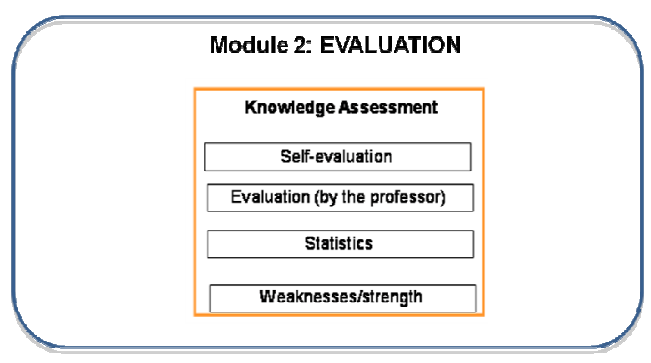

Figure 3: Evaluation module.

The second module (Figure 3 ) compiles all entry information by the user and results from module 1 in order to establish topic review guidelines that will help the student in his or her self-evaluation, to allow the professor evaluate the strengths and weaknesses of the student and to provide statistical data that will help assess the effectiveness of this pedagogical tool. This will also be helpful in the context of continuously updating and improving the operational characteristics and user-interface features of the pedagogical tool.

DISCUSSION

We expect this pedagogical tool to contribute to the motivation of students for analog electronics, since it has been demonstrated that students find that the blended learning resulting from using eLearning techniques is a valuable enhancement to their studies [1]. Therefore, we anticipate that students will be motivated to use this online tool to review key topics in analog electronics when the opportunities for their application arise (e.g. while taking a more systems-oriented course such as linear feedback analysis or analog filter design, which rely more on transfer functions based systems analyses than circuits theory) during the interruption periods between the circuits oriented analog electronics courses. To enhance the formative auto-evaluation aspect of this pedagogical tool, the diagnostic functions related to each object in module 1 (Figure 2) as well as feedback for overcoming weak points at the end of each online session will be made clear to the students (as recommended in [2]) thanks to the interaction with module 2 (Figure 3). This will be strengthened by the inclusion of circuit oriented objects (Figure 2) that are directly applicable to specific concepts covered in such systems-oriented courses. Moreover, the participation of students in the development of this tool and in publicizing its purpose and usefulness within the student community will be beneficial to this project.

\section{REFERENCES}

1. Uren M, Uren J. "eTeaching and eLearning to enhance learning for a diverse cohort in engineering education," Engineering Education, vol. 4, no. 2, 2009.

2. Dopper SM, Sjoer E. "Implementing formative assessment in engineering education: the use of the online assessment system Etude," European Journal of Engineering Education, vol. 29, no. 2, June 2004. 